\title{
菊糖分子量的高效凝胶过滤色谱检测方法及其在 贮藏过程中的变化研究
}

\author{
李琬聪 ${ }^{(2)}$ ，李青 ${ }^{(1)}$, 董方 ${ }^{(1)}$, 郭占勇 ${ }^{(\mathbb{*}}$ \\ (1) 中国科学院烟台海岸带研究所, 海岸带生物学与生物资源利用重点实验室, 烟台 264003; \\ (2) 中国科学院大学, 北京 100049 \\ * 联系人, E-mail: zhanyongguo@hotmail.com
}

收稿日期: 2016-02-11; 接受日期: 2016-03-26; 网络版发表日期: 2016-08-26

“十二五”国家科技支撑计划(批准号: 2012BAD32B09)资助

\begin{abstract}
摘要菊芋是一种耐盐抗旱植物, 可用于生产菊糖. 菊糖具有良好的生物活性, 其活性受到聚合度的影响. 本 研究对比了各项条件, 总结得到了一套可以用于测定菊糖相对分子量的高效凝胶过滤色谱(HPGFC)方法: 纯水 为流动相, $0.8 \mathrm{~mL} / \mathrm{min}$ 洗脱, Shodex OHpak SB-804HQ 色谱柱检测, 柱温控制在 $30^{\circ} \mathrm{C}$, 样品进样量为每次 $20 \mu \mathrm{L}$, 检测器为示差折光检测器 (RID), 采用 Sigma 公司生产的右旋糖酐标准品, 绘制二次方的标准曲线, 检测菊糖的相对分子质量. 同时利用确定的凝胶过滤色谱(GFC)方法, 观察了种植于海岸带盐碱地的菊芋块茎 采收后在贮藏过程中的菊糖分子量的变化, 发现在 $-20^{\circ} \mathrm{C}$ 的贮藏条件下, 菊芋中的菊糖不会发生剧烈降解, 可长期保存提取分子量相近的菊糖. 本研究对分析菊糖的分子性质提供了方法基础, 对菊芋的采收贮藏有指 导意义.
\end{abstract}

关键词 HPGFC，菊糖，相对分子质量，贮藏条件

菊芋(Jerusalem artichoke)又名洋姜、鬼子姜, 是 一种菊科向日葵属多年生草本植物, 它耐寒抗冻, 抗 贫㾑和干旱, 对土壤要求不高, 抗风沙和病虫害, 适 应性强, 繁殖能力强, 栽植一次, 可连续多年利用 ${ }^{[1]}$. 因此, 它可以不与其他作物竞争土地资源. 菊芋中主 要含有 70\% 80\%的水分、15\% 20\%的菊糖以及少量 的蛋白质、灰分等其他物质 ${ }^{[2]}$. 因此, 在海岸带周边 盐碱地种植菊芋, 不仅可以有效提高荒地利用率, 还
可以得到具有工业生产价值的菊糖产品.

菊糖是由 $\mathrm{D}$-果糖经 $\beta(2 \rightarrow 1)$ 键连接而成的线性 直链多糖, 末端常带有一个葡萄糖残基 ${ }^{[3]}$. 由于 其特殊的 $\beta(2 \rightarrow 1)$ 糖苷键结构, 菊糖在口腔、胃、 小肠中均不会发生消化分解, 只能在结肠中被双 歧杆菌等部分发酵分解产生少量热量 ${ }^{[3 \sim 5]}$. 菊糖作 为一种天然的功能性多糖, 具有改善肠道微环境、 调控血脂和血糖水平、预防肥胖症、促进矿物质

引用格式: 李琬聪, 李青, 董方, 等. 菊糖分子量的高效凝胶过滤色谱检测方法及其在尖藏过程中的变化研究. 中国科学: 生命科学, 2016, 46: 1107-1112 Li W C, Li Q, Dong F, et al. Inulin molecular detection methods of high-performance gel filtration chromatography and changes in inulin molecular weight during storage. Sci Sin Vitae, 2016, 46: 1107-1112, doi: 10.1360/N052016-00059 
吸收以及抗氧化和抗癌等突出生理功能 ${ }^{[1]}$. 菊糖 的性质使得它成为一种健康的食品添加剂、低热 量甜味剂、唖食纤维和一种脂肪替代品 ${ }^{[6]}$.

菊糖聚合度在 2 100 变化. 分子的链长、组成等 结构性质与获取菊糖的植物的种类、采收时间、提取 和提取后处理过程有关 ${ }^{[7]}$. 菊糖聚合度的变化对菊芋 和菊糖的工业应用和价值有很大影响 ${ }^{[8]}$. 然而, 对于 菊糖聚合度的检测方法目前尚无系统研究.

高效凝胶过滤色谱 (high performance gel filtration chromatography, HPGFC)也称高效凝胶渗透 色谱 (high performance gel permeation chromatography, HPGPC) 或高效体积排阻色谱 (highperformance size exclusion chromatography, HPSEC), 具有高效、快速、灵敏度高等特点, 其使用的色谱 柱表现出在一定相对分子量范围内分配系数 $\mathrm{Kd}$ 与相对分子量对数呈一定的相关性 ${ }^{[9]}$, 已经逐渐 被认为是研究多种聚合物相对分子量及相对分子 量分布的最有效的手段之一, 近年来, 逐渐开始 广泛应用于多糖、树脂等多种聚合物的分子量 测定 ${ }^{[10]}$.

本文对比各项条件, 总结得到了一套可以用于 测定了菊糖相对分子质量的 HPGFC 方法, 并且利用 GFC 方法得到的标准曲线检测了种植于海岸带盐碱 地的菊芋块茎采收后在败藏过程中的菊糖分子量的 变化.

\section{1 材料与方法}

\section{1 材料与试剂}

菊芋样品采收自山东省东营市垦利县(位于黄 河三角洲, N $37^{\circ} 24^{\prime} \sim 38^{\circ} 10^{\prime}$, E $118^{\circ} 15^{\prime} \sim 119^{\circ} 19^{\prime}$ ). 唇 利县气候为夏季炎热多雨, 冬季干旱寒冷, 降水时空 分布不均; 土壤质地偏轻, 养分贫乏, 盐碱较重, 在 表层中, 某些地方的盐的质量分数甚至会高于 $15 \mathrm{~g} / \mathrm{kg}^{[11]}$.

实验所用试剂氢氧化钙、磷酸、 $30 \%$ 双氧水、苯 酚、硫酸和 3,5-二硝基水杨酸等皆为分析纯, 超纯水 (18.2 M $\Omega$ resistivity)由 Milli-Q Element 水纯化系统制 备. 右旋糖酐标准品两组, 分别采购自 SigmaAldrich(美国)和 APSC 公司(美国).

HPGFC 所用高效液相为安捷伦公司的 Agilent
$1260 \operatorname{HPLC}$ (美国), 配置为四元泵、手动进样器、柱 温箱和示差折光检测器 (refraction index detector, RID).

\section{2 菊糖样品制备工艺流程}

根据查阅的文献及专利 ${ }^{[12,13]}$, 确定了如下实验 方案:

(i) 菊芋样品切片, 煮沸 5 min 除去多酚氧化酶 (polyphenol oxidase, PPO), $60^{\circ} \mathrm{C}$ 下干燥 $8 \mathrm{~h}$, 粉碎.

(ii) 加入 15 倍于粉碎样重量的水, $90^{\circ} \mathrm{C}$ 下浸提 $40 \mathrm{~min}$, 提取两次.

（iii）浸提液浓缩后, 加入氢氧化钙乳液调节 $\mathrm{pH}$ 约为 11 , 放入 $80^{\circ} \mathrm{C}$ 水浴中保温 $1 \mathrm{~h}$, 用纱布抽滤两次 (第 1 次 2 层纱, 第 2 次 4 层纱), 滤液加少量磷酸调 $\mathrm{pH}$ 至 $8,1789 \times g$ 离心 $7 \mathrm{~min}$.

(iv) 离心液加入 $2.5 \% \sim 3.0 \%$ 的 $\mathrm{H}_{2} \mathrm{O}_{2}, 40^{\circ} \mathrm{C}$ 脱色 40 min.

（v) 脱色后的溶液浓缩至尽量少，加入 $4 \sim 5$ 倍 体积的无水乙醇沉淀, 静置后抽滤, 滤液再浓缩后加 乙醇沉淀, 重复至无沉淀产生(一般需重复 3 4 次).

(vi) 冷冻干燥沉淀, 得白色或乳白色菊糖.

按照以上流程, 分别提取了新鲜菊芋中的菊糖 以及陉藏于 $-20^{\circ} \mathrm{C}$ 下 $60,80,120,140,180$ 天后的菊芋 中的菊糖.

\section{3 色谱条件的选择}

凝胶过滤色谱 (gel filtration chromatography, $\mathrm{GFC}$ ) 是根据分子大小不同, 在填料中经过的路径不 同，被淋洗出的时间不同来进行分离的.

GFC 计算样品分子量的方法是：检测不同分子 量的标准品, 以出峰时间为横坐标, $\log \mathrm{Mw}$ 为纵坐标 做标准曲线，根据检测样品的出峰时间计算样品分 子量.

（1）流速的选择. 采用纯水为洗脱剂, 选择 RID 检测, 柱温控制在 $30^{\circ} \mathrm{C}$, 分别采用 0.6 和 $0.8 \mathrm{~mL} / \mathrm{min}$ 的流速检测样品.

(2) 标准品的选择. 标准曲线的绘制需要选择 合适的标准品. 对比了两家公司生产的不同分子量 的右旋糖酐标准品绘制的标准曲线在测试菊糖分子 量时的准确度, 分别是 Sigma-Aldrich 公司生产的右 旋糖䣶标准品(分子量 $\mathrm{Mw}$ 分别为 1000, 5000, 12000, 25000 和 $50000 \mathrm{Da}$ )和 APSC 公司生产的右旋糖酐标 
准品(分子量 $\mathrm{Mw}$ 分别为 $180,505,1200,3650$ 和 20100 Da). 分析过程 HPLC 运行条件为: $0.8 \mathrm{~mL} / \mathrm{min}$ 纯水 洗脱, 柱温 $30^{\circ} \mathrm{C}$, RID 检测出峰, 样品进样量为每次 $20 \mu \mathrm{L}$.

标准曲线准确度的测定方法: 制备 5 份 $10 \mathrm{mg} / \mathrm{mL}$ 右旋糖䣶样品, 按上述色谱条件分别进样 分析, 通过 HPGFC 法计算得到的相对分子质量与标 准品已知相对分子质量相对照, 由相对误差衡量标 准曲线准确度. 以标准曲线的准确度为衡量指标, 选 择合适的标准品.

(3) 色谱柱的选择. 选取了两根不同的色谱柱 进行检测, 分别是 TSK G3000PW $\mathrm{XL}_{\mathrm{XL}}$ 色谱柱和 Shodex OHpak SB-804HQ 色谱柱. 同样采用标准曲线准确度 为衡量标准, 选择合适的色谱柱. 标准曲线准确度测 定方法见(2).

(4) 标准曲线拟合形式的选择. 根据凝胶过滤 色谱的工作原理, 推断标准曲线应该采用三次方程 的形式拟合, 但在实际应用中, 会出现其他幂次的方 程更适用的情况, 因此考察了一次方程到五次方程 的拟合形式. 采用标准曲线准确度为衡量标准, 选择 合适的拟合形式. 标准曲线准确度测定方法见(2).

\section{4 菊糖相对分子量的测定}

(1) 标准曲线的绘制. 分别精确称取一定量的 右旋糖酐标准品, 用超纯水溶解, 配成 $10.0 \mathrm{mg} / \mathrm{mL}$
的标准品溶液. 经 1.3 中各项比较后, 确定了合适的 色谱条件, 在此条件下分别进样, 测得各色谱峰保留 时间. 以不同分子量标准右旋糖酐的保留时间 (retention time, RT) 为横坐标, 相应的相对分子质量 对数值 $(\mathrm{lgMw})$ 为纵坐标, 绘制标准曲线.

(2) 菊糖样品分子量的测定. 分别称取提取得 到的菊糖, 用超纯水溶解, 配成 $10.0 \mathrm{mg} / \mathrm{mL}$ 的样品 溶液. 按制作标准曲线的色谱条件进样, 得到菊糖的 保留时间, 根据标准曲线计算菊糖相对分子质量.

\section{2 结果与分析}

\section{1 色谱条件的确定}

(1) 流速的确定. 流速对样品的分离有很大影 响, 因此, 分别采用了 0.6 和 $0.8 \mathrm{~mL} / \mathrm{min}$ 的流速检测 了同一份菊糖样品, 图 1 是流速分别为 0.6 和 $0.8 \mathrm{~mL} / \mathrm{min}$ 时样品的出峰情况, 对比发现, 二者峰形 变化不大, 仅出峰时间略有不同, 流速为 $0.8 \mathrm{~mL} / \mathrm{min}$ 时样品会更早出峰, 考虑到效率问题, 选择 0.8 $\mathrm{mL} / \mathrm{min}$ 的流速进行后续的检测.

(2) 标准品的确定. 不同的标准品绘制的标 准曲线不同, 对菊糖样品分子量的检测有较大影响. 因此, 以不同标准品绘制的标准曲线准确度为衡量 标准, 检测结果如表 1 所示. 对比发现, 采用 Sigma

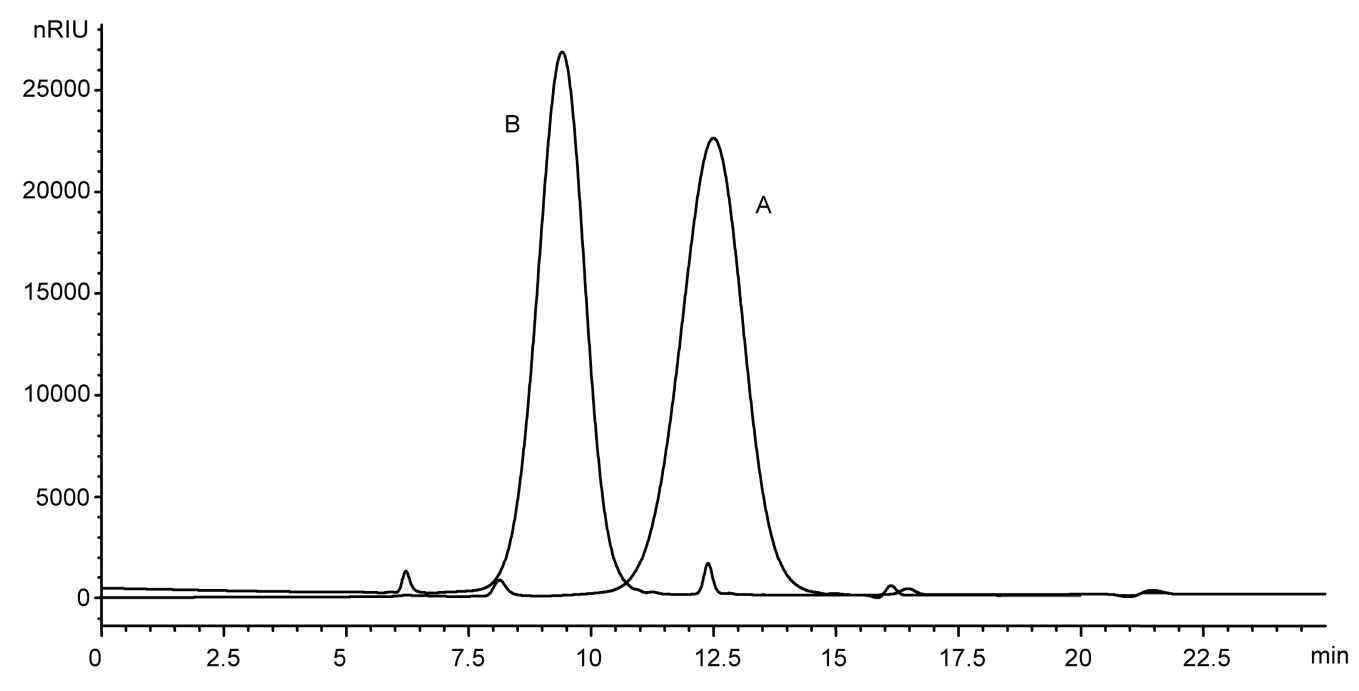

图 1 不同流速洗脱同一样品的色谱图

A: 流速为 $0.6 \mathrm{~mL} / \mathrm{min}$ 时的样品谱图; B: 流速为 $0.8 \mathrm{~mL} / \mathrm{min}$ 时的样品谱图 
公司生产的右旋糖䣲做标准品绘制的标准曲线计算 出的相对分子质量更接近已知的值，其相对误差更 小, 故采用 Sigma 公司生产的右旋糖䣶(分子量 $\mathrm{Mw}$ 分别为 $1000,5000,12000,25000$ 和 $50000 \mathrm{Da}$ )做标 准品.

(3) 色谱柱的确定. 不同色谱柱对菊糖样品的 检测效果不同, 为选择合适的色谱柱, 以不同的色谱 柱绘制的标准曲线的准确度为衡量标准, 检测结果 见表 2. 对比数据可以看出, Shodex OHpak SB804HQ 色谱柱更适合用于测量菊糖的相对分子质量.

(4) 标准曲线拟合形式的确定. 不同拟合形 式拟合出的标准曲线计算得到的菊糖样品分子量不 同, 因此，同样以标准曲线准确度为衡量标准来选择 合适的标准曲线拟合形式. 一次到三次方程拟合形 式的标准曲线的准确度见表 3. 四次方程和五次方程 的标准曲线如图 2 所示, 在待测的分子量范围内出现 了斜率为负值的情况，在此种情况下，曲线无法用来 计算相对分子质量, 故四次方程和五次方程不适合 作为标准曲线计算菊糖分子量.

根据数据对比发现，采用二次方程的形式拟合

表 1 不同公司生产的右旋糖䣶标准品绘制的标准曲线准 确度 $(\%)$

\begin{tabular}{ccc}
\hline 编号 & Sigma 公司标准品 & APSC 公司标准品 \\
\hline 1 & 4.58 & 12.17 \\
2 & 5.98 & 12.58 \\
3 & 5.64 & 11.67 \\
4 & 5.10 & 12.17 \\
5 & 4.74 & 12.58 \\
\hline
\end{tabular}

表 2 不同色谱柱绘制的标准曲线的准确度 $(\%)$

\begin{tabular}{ccc}
\hline 编号 & $\begin{array}{c}\text { Shodex OHpak SB-804HQ } \\
\text { 色谱柱 }\end{array}$ & TSK G3000PW $_{\mathrm{XL}}$ 色谱柱 \\
\hline 1 & -0.42 & -1.62 \\
2 & 0.98 & -1.62 \\
3 & 0.64 & -2.06 \\
4 & 0.10 & -1.92 \\
5 & -0.24 & -1.76 \\
\hline
\end{tabular}

表 3 不同拟合形式的标准曲线的准确度 $(\%)$

\begin{tabular}{cccc}
\hline 编号 & 一次方程 & 二次方程 & 三次方程 \\
\hline 1 & -14.34 & -0.42 & 4.58 \\
2 & -13.32 & 0.98 & 5.98 \\
3 & -13.58 & 0.64 & 5.64 \\
4 & -13.96 & 0.10 & 5.10 \\
5 & -14.22 & -0.24 & 4.74 \\
\hline
\end{tabular}
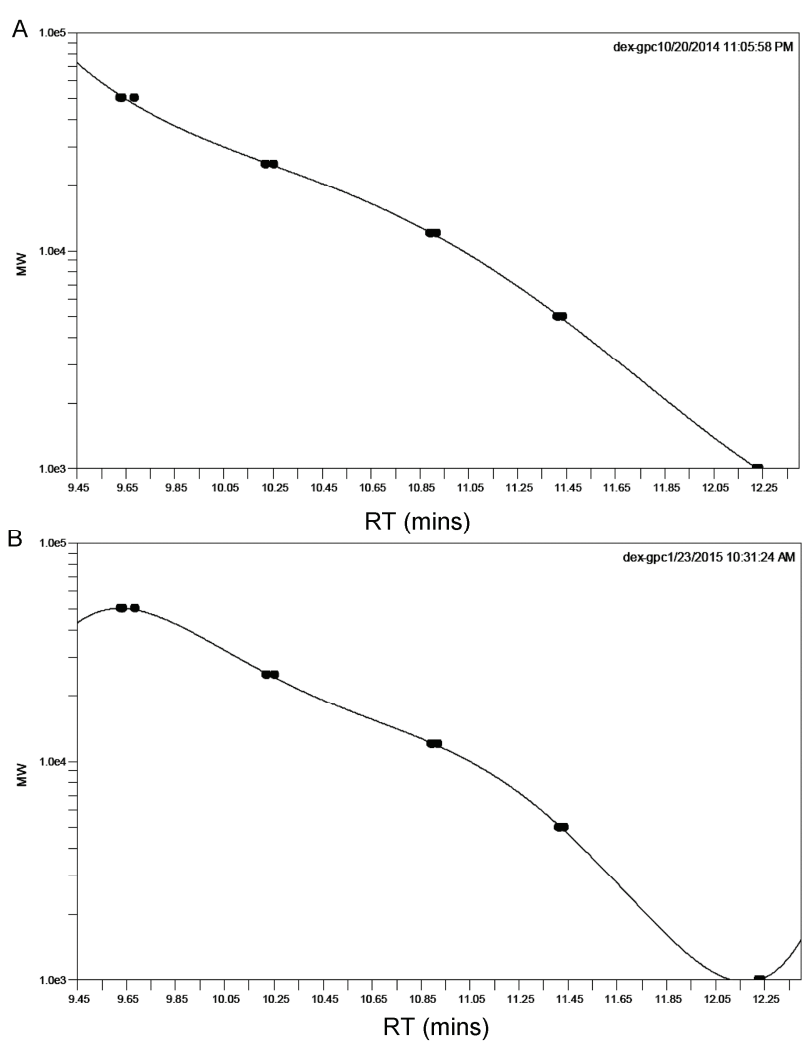

图 2 标准曲线

A：四次方形式拟合的标准曲线; B: 五次方形式拟合的标准曲线

出的标准曲线计算出的相对分子质量其相对误差最 小，而并非根据原理推断出的三次方程的拟合形式， 故后续工作采用二次方程拟合的标准曲线进行计算, 标准曲线方程为: $y=-2.405+1.827 x-0.113 x^{2}$.

综合以上各项条件的对比，最终得到了一套 适用于菊糖分子量测定的 HPGFC 方法: 纯水为流 动相, $0.8 \mathrm{~mL} / \mathrm{min}$ 洗脱, Shodex OHpak SB-804HQ 色 谱柱检测, 柱温控制在 $30^{\circ} \mathrm{C}$, 样品进样量为每次 $20 \mu \mathrm{L}$, 检测器为 RID, 采用 Sigma 公司生产的右旋 糖䣶标准品, 绘制二次方的标准曲线, 检测菊糖的相 对分子质量.

\section{2 贮藏过程中菊糖相对分子量的变化}

图 3 是在 $-20^{\circ} \mathrm{C}$ 的聍藏条件下, 菊芋中菊糖分子 量的变化. 可以看出, 在 $-20^{\circ} \mathrm{C}$ 下栬藏菊芋几乎不会 引起菊糖的链长改变. 聍藏 120 天, 菊芋中菊糖的分 子量依然维持在 $3500 \mathrm{Da}$ 左右. 当败藏到 140 天时, 菊糖的分子量出现一个较为明显的下降, 降至

1110 


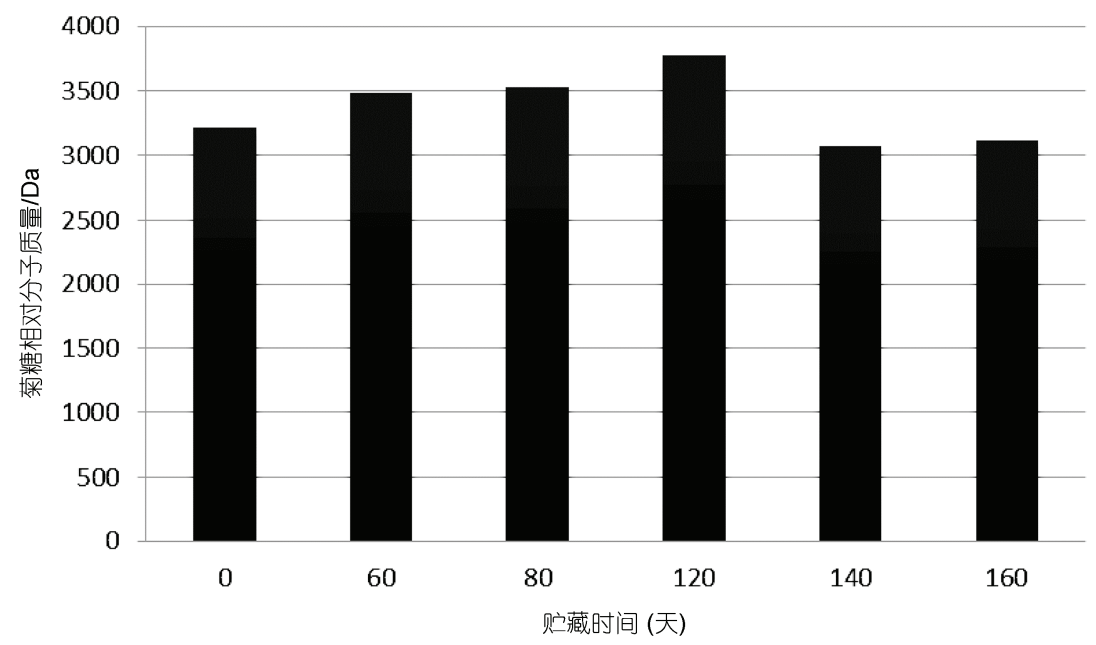

图 3 贮藏过程中菊糖分子量的变化

$3000 \mathrm{Da}$ 左右. 当聍藏至 160 天时, 菊糖的分子量依 然为 $3000 \mathrm{Da}$ 左右, 不再发生明显变化. 由此表明在 $-20^{\circ} \mathrm{C}$ 的聍藏条件下, 菊芋中菊糖可以在 120 天内不 发生剧烈的降解而使得分子量减小, 但之后会有一 个短暂的降解过程, 分子量减小, 聚合度降低 2 3 左 右, 然后菊芋中的菊糖会继续处在一个较为稳定的 状态, 不再发生剧烈的降解. 这对于菊芋采收后的咜 藏条件和时限有很好的指导作用.

\section{3 讨论}

本研究对比了各项条件, 总结得到了一套可以 用于测定菊糖相对分子量的 HPGFC 方法: 纯水为流
动相, $0.8 \mathrm{~mL} / \mathrm{min}$ 洗脱, Shodex OHpak SB-804HQ 色 谱柱检测, 柱温控制在 $30^{\circ} \mathrm{C}$, 样品进样量为每次 $20 \mu \mathrm{L}$, 检测器为 RID, 采用 Sigma 公司生产的右旋 糖䣶标准品, 绘制二次方的标准曲线, 检测菊糖的相 对分子质量.

利用确定的 GFC 方法，检测了种植于海岸带盐 碱地的菊芋块茎采收后在咜藏过程中的菊糖分子量 的变化, 发现在 $-20^{\circ} \mathrm{C}$ 的败藏条件下, 菊芋中的菊糖 不会发生剧烈降解，可长期保存提取分子量相近的 菊糖.

本研究为分析菊糖的分子性质提供了方法基础, 对菊芋的采收以及菊糖的的加工和只藏有十分重要 的意义.

\section{参考文献}

1 曾小宇, 罗登林, 刘胜男, 等. 菊糖的研究现状与开发前景. 中国食品添加剂, 2010, 4: 222-227

2 魏凌云. 菊粉的分离纯化过程和功能性产品研究. 博士学位论文. 杭州: 浙江大学, 2006

3 Causey J L, Feirtag J M, Gahaher D D, et al. Effects of dietary inulin on serum lipids, blood glucose and the gastrointestinal environment in hypercholesterolemic men. Nutr Res, 2000, 20: 191-201

4 Bach V, Jensen S, Clausen M R, et al. Enzymatic browning and after-cooking darkening of Jerusalem artichoke tubers (Helianthus tuberosus L.). Food Chem, 2013, 141: 1445-1450

5 Li L L, Li L, Wang Y, et al. Biorefinery products from the inulin-containing crop Jerusalem artichoke. Biotechnol Lett, 2013, 35: 471-477

6 熊政委, 董全. 菊糖的生理功能和在食品中应用的研究进展. 食品工业科技, 2012, 33: 351-353

7 Ronkart S N, Blecker C S, Fourmanoir H, et al. Isolation and identification of inulooligosaccharides resulting from inulin hydrolysis. Anal Chim Acta, 2007, 604: 81-87

8 Roberfroid M B. Introducing inulin-type fructans. Brit J Nutr, 2005, 93: S13-S25

9 成跃祖. 凝胶渗透色谱法的进展及其应用. 北京: 中国石化出版社, 1993.10-78

10 岳莉, 董安石, 叶文, 等. 高效凝胶渗透色谱法测定沥青树脂的相对分子量及其分布. 辽宁科技大学学报, 2011, 34: 141-143 


\title{
Inulin Molecular Detection Methods of High-Performance Gel Filtration Chromatography and Changes in Inulin Molecular Weight During Storage
}

\author{
LI WanCong ${ }^{1,2}$, LI Qing ${ }^{1}$, DONG Fang ${ }^{1} \&$ GUO ZhanYong ${ }^{1 *}$ \\ 1 Key Laboratory of Coastal Biology and Bioresource Utilization, Yantai Institute of Coastal Zone Research, Chinese Academy of Sciences, Yantai \\ 264003, China; \\ 2 University of Chinese Academy of Sciences, Beijing 100049, China;
}

\begin{abstract}
Jerusalem artichoke is a salt- and drought-resistant plant used for the production of inulin. Inulin has good biological activity, which is affected by its degree of polymerization. In this study, we compared various conditions for standardizing a high-performance gel filtration chromatography (HPGFC) method that can be used to determine the relative molecular weight of inulin. A Shodex ${ }^{\mathrm{TM}}$ GPC OHpak SB-804 column was used, with ultrapure water as the mobile phase at a flow rate of $0.8 \mathrm{~mL} / \mathrm{min}$. The column temperature was $30^{\circ} \mathrm{C}$ and the injection volume was $20 \mu \mathrm{L}$. A differential refractive index detector was used for detection, and dextran standard purchased from Sigma-Aldrich ${ }^{\circledR}$ was used as the standard. The standard curve was fit with a quadratic equation to determine the relative molecular weight of inulin. Furthermore, we studied the effect of storage on the molecular weight of inulin. Inulin extracted from Jerusalem artichoke did not degrade sharply under storage conditions of $-20^{\circ} \mathrm{C}$. This knowledge could be used to preserve Jerusalem artichoke to get inulin with similar molecular weight. Our work provides a method to analyze the molecular weight of inulin, and it is instructive for the harvesting and preservation of Jerusalem artichoke.
\end{abstract}

HPGFC, inulin, relative molecular weight, storage conditions

doi: 10.1360/N052016-00059 